\title{
Editorial
}

\section{Erythrocytes: Death of a mummy}

\author{
E Daugas ${ }^{1}$, C Candé ${ }^{1}$ and G Kroemer ${ }^{\star, 1}$ \\ ${ }^{1}$ Centre National de la Recherche Scientifique, UMR1599, Institut Gustave \\ Roussy, 39 rue Camille-Desmoulins, F-94805 Villejuif, France \\ *Corresponding author: G Kroemer, CNRS-UMR1559, Institut Gustave Roussy, \\ Pavillon de Recherche 1, 39 rue Camille-Desmoulins, F-94805 Villejuif, France, \\ Tel: 33-1-42 1160 46; Fax: 33-1-42 1160 47; \\ E-mail: kroemer@igr.fr
}

Simple spirits consider mammalian erythrocytes as membrane-surrounded hemoglobin-containing vesicles floating in circulation. Indeed, they lack organelles including nuclei and mitochondria and, in a sense, they cannot be considered as true cells. Nonetheless, erythrocytes have been intriguing researchers interested in apoptosis.

The red blood cell (RBC) life cycle can be divided in two phases, the first one, erythopoiesis consisting in the production of mature erythrocytes in hematopoietic organs, the second one concerning the proper existence of the mature eythrocyte in circulation. Erythropoiesis results from the progressive differentiation of erythroid progenitors, from proerythroblasts through polychromatophylic erythroblasts, then orthochromatic erythroblasts to mature erythrocytes. It is now well established that the hormone erythropoetin stimulates erythropoiesis, at least in part, by protecting erythroblasts from apoptosis presumably by upregulating the anti-apoptotic protein $\mathrm{Bcl}-\mathrm{X}_{\mathrm{L}} .^{1,2}$ Indeed, conditional knock-out of $\mathrm{Bcl}-\mathrm{X}_{\mathrm{L}}$ suppresses erythropoiesis at a late stage resulting in hemolytic anemia, ${ }^{3}$ and erythropoietin withdrawal triggers erythroblast death and caspase activation. ${ }^{4}$ A CD95- and caspase-dependent negative regulation of erythropoiesis has been described at the local level. Orthochromatic erythroblasts expressing CD95 ligand would stimulate the CD95 death receptor expressed on immature erythroblasts, thereby creating a negative feedback loop controlling erythropoiesis. Erythopoietin would antagonize this negative control. ${ }^{5,6}$ Thus, not surprisingly, erythrocyte precursors, which are true organelle-containing cells, are susceptible to apoptosis induction, including in pathological stages such as $\beta$-thalassemia major, ${ }^{7}$ idiopathic acquired sideroblastic anemia ${ }^{8}$ and parvovirus B19 infection. ${ }^{9}$

Surprisingly, caspase activation has not only negative effects on erythropoiesis. Rather, it appears that the activation of effector caspases is required for $\mathrm{RBC}$ formation. ${ }^{10}$ In vitro erythropoiesis reportedly is blocked by the pan-caspase inhibitor Z-VAD.fmk. During erythroblast differentiation caspases-3, -6 and -7 are proteolytically activated and cleave nuclear proteins (lamin $B$ and acinus) yet leave intact the transcription factor GATA-1 and fail to trigger the exposure of phosphatidylserine on the plasma membrane surface. ${ }^{10}$ This suggests that a sort of abortive apoptosis participates in the differentiation and enucleation of erythroid cells. However, caspase- $3^{-1-}$ mice do not exhibit any obvious defect in erythropoiesis, and transgenetargeted overexpression of $\mathrm{Bcl}-2$ in the erythroid lineage does not induce any erythropoietic disorder in vivo. ${ }^{11}$ Further studies will thus have to confirm the implication of caspase activation and abortive apoptosis in erythroid differentiation. Intriguingly, Nagata and co-workers recently reported that DNAse II is required for erythropoiesis in the fetal liver. According to these authors, DNAse II present in macrophages would be required for efficient enucleation of eythrocyte precursors. DNAse II thus would not participate in a cell-autonomous process and rather act in the heterophagic removal of the nucleus of the differentiating erythroblast, ${ }^{12}$ much in the way as this has been described for the macrophage-mediated removal of apoptotic thymocytes. $^{13}$

Mammalian fetal erythrocytes as well as erythrocytes from amphibians, reptiles and birds do contain a nucleus, and these cells can undergo normal apoptosis. Thus, fetal human erythrocytes circulating in maternal blood undergo apoptosis in situ. ${ }^{14}$ Moreover, chicken erythrocytes can be driven into apoptosis (nuclear and cellular shrinkage, chromatin condensation, TUNEL positivity, staining with Annexin-V) by incubating them either in the absence of serum or in the presence of staurosporin plus cycloheximide. ${ }^{15}$ This process is not prevented by Z-VAD.fmk and is not accompanied by caspase activation, suggesting that chicken erythrocyte apoptosis occurs in a caspaseindependent fashion.

Two fascinating papers published in this issue of CDD now tackle the question whether mature human erythrocytes can undergo apoptosis-like cell death. ${ }^{16,17}$ In contrast to true cells, human erythrocytes fail to die in the absence of serum or upon addition of staurosporin+cycloheximide, in vitro. However, RBC life span is limited to approximately 120 days in vivo. It has been widely assumed that RBC aging would lead to an increase in intracellular calcium, modifications of the erythrocyte shape, protein degradation and externalization of phosphatidylserine, thereby activating a clearance mechanism involving heterophagic removal in the reticuloendothelial system. ${ }^{18,19}$ Indeed, splenectomy is accompanied by the occurrence of aged erythrocytes in peripheral blood that have failed to be cleared.

Here, the groups headed by Klaus Schulze-Osthoff ${ }^{16}$ and the tandem formed by Jean-Claude Ameisen and Jean Montreuil ${ }^{17}$ report the intriguing finding that $\mathrm{Ca}^{2+}$ ionophores (A23187 or ionomycin) induce an in vitro erythrocyte senescence process characterized by cell shrinkage, membrane microvesiculation, and phosphatidylserine externalization. This process culminates in erythrocyte disintegration, phagocytosis in the presence of macrophages in vitro, and in clearance from the circulation in vivo. 


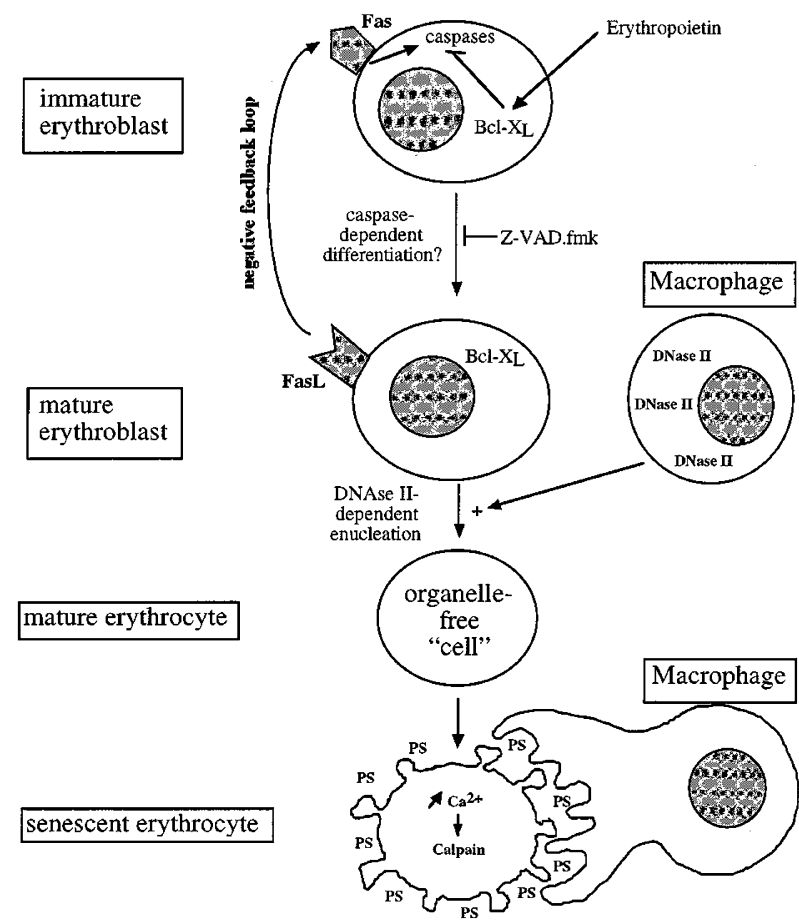

Figure 1 Erythroblasts, the erythrocyte precursors are organelle-containing cells which can undergo full-blown apoptosis. The differentiation process may involve a caspase-dependent step and successfull enucleation requires the concourse of DNAse II expressing macrophages. Mature, organelle-free erythrocytes can be driven into in vitro senescence by addition of external $\mathrm{Ca}^{2+}$ or addition of $\mathrm{Ca}^{2+}$ ionophore, which triggers $\mu$-calpain activation and $\mu-$ calpain-dependent changes in cellular shape and plasmamembrane structure (in particular PS exposure) reminescent of apoptosis.

Surprisingly, in vitro senescence is not just due to a disruption of ion homeostasis and rather involves the activation of proteolytic enzymes, as indicated by the fact that cysteine protease inhibitors such as Ac-DEVD-CHO or leupeptin prevent all the features of $\mathrm{Ca}^{2+}$ ionophoreinduced eythrocyte senescence. Erythrocytes were found to contain pro-caspase-3 and -8 as well as $\mu$-calpain. Upon $\mathrm{Ca}^{2+}$ exposure, $\mu$-calpain is cleaved to its active fragments which in turn are likely responsible for the degradation of spectrin (erythrocyte fodrin). In contrast, neither procaspase-3 nor pro-caspase-8 become activated in erythrocytes, which can be explained by the absence of two essential apoptosome components, Apaf-1 and cytochrome $c$. Thus, calpain but not caspases participate in erythrocyte senescence, and the effect of Ac-DEVD-CHO must be attributed to the inhibition of another protease (presumably $\mu$-calpain) than its classical target, caspase-3.

Calpain has also been involved in apoptotic signaling in true nucleate cells, according to numerous reports. For instance, pharmacological inhibition of calpains can prevent cell death. This applies to excitotoxin-induced neuronal cell death, ${ }^{20}$ hypoxia-ischemia damage of the brain, ${ }^{21}$ germ cell death in experimental testicular torsion, ${ }^{22}$ neutrophil apoptosis triggered by cycloheximide, ${ }^{23}$ and radiation-induced apoptosis. ${ }^{24}$ Transfection with the endogenous calpain inhibitor calpastatin can render B cells resistant to B cell receptor-induced apoptosis. ${ }^{25}$ Calpains are activated by increased cytosolic $\mathrm{Ca}^{2+}$ levels and they trigger apoptosis by proteolytically activating caspases (in particular caspases-3, -9 and -12$)^{26,27}$ and/or by cleaving members of the $\mathrm{Bcl}-2$ family including $\mathrm{Bcl}-2,{ }^{28} \mathrm{Bcl}-\mathrm{X}_{\mathrm{L}}$ (which convert from an antiapoptotic to a pro-apoptotic function), $\mathrm{Bax}^{29}$ and $\mathrm{Bid}^{30}$ (which upon truncation increase their apoptogenic potential). Numerous reports underline the existence of a cross-talk of mutual activation between caspases and calpains, and growing evidence supports the notion that either classes of proteases can substitute for each other in apoptotic pathways. ${ }^{31}$ Knock-out of $\mu$-calpain reveals a deficiency in platelet aggregation, ${ }^{32}$ in accord with the observation that $\mathrm{Ca}^{2+}$ ionophore stimulation of platelets induces calpaindependent and caspase-independent apoptosis-like events including phosphatidylserine exposure. ${ }^{26}$

A semantic and hence delicate problem arises from the use of erythrocytes for the study of cell death or apoptosis. If we consider $\mathrm{Ca}^{2+}$ ionophore-induced RBC senescence as 'apoptotic', then the results reported here ${ }^{16,17}$ may be interpreted to mean that 'apoptosis' can occur in the absence of mitochondria, nuclei, and caspase activation. That apoptosis may occur in the absence of a nucleus (that is in freshly enucleated cells, so-called cytoplasts) or in the absence of caspase activation (that is in the presence of caspase-inhibitors or in Apaf-1 or caspase knock-out cells) has been reported in the past, ${ }^{33-35}$ and the true originality of the present articles ${ }^{16,17}$ would be to show death in the absence of mitochondria. ${ }^{36}$ However, in a way the erythrocyte must be considered as a post-apoptotic creature, in the sense that transient, presumably mitochondrion-triggered caspase activation ${ }^{10}$ has lead to the formation of an organelle-free mummy (just as ancient Egyptians removed organs from the corpse before mummification). Seen from this angle, it appears logical that the final implosion of RBC occurs via a non-apoptotic process which we might call erythroptosis.

Irrespective of this semantic problem, the second death of erythrocytes is characterized by some features that are shared by apoptosis. Indeed, classical apoptosis of mammalian cells, in vitro erythrocyte senescence, ${ }^{16,17}$ and artifactual induction of cell death in monocellular organisms ${ }^{37,38}$ is invariably accompanied by phosphatidylserine exposure and microvesiculation as well as nuclear condensation (when a nucleus is present). Again, two interpretations of this parallelism are possible. Either it hints at the existence of a common biochemical 'program' that has been conserved throughout evolution, or it simply means that an increase in entropy leading to disintegration of a cell or a cell-derived vesicle gives rise to a common phenomology.

\footnotetext{
1. Socolovsky M, et al. (1999) Cell 98: 181-191

2. Motoyama N, et al. (1999) J. Exp. Med. 189: 1691-1698

3. Wagner KU, et al. (2000) Development 127: 4949-4958

4. Gregoli PA and Bondurant MC. (1999) J. Cell Physiol. 178: 133-143

5. De Maria R, et al. (1999) Nature 401: 489-493

6. De Maria R, et al. (1999) Blood 93: 796-803
} 
7. Mathias LA, et al. (2000) Exp. Hematol. 28: 1343-1353

8. Matthes TW, et al. (2000) Br. J. Haematol. 111: 843-852

9. Yaegashi N, et al. (1999) J. Infect. 39:68-76

10. Zermati $Y$, et al. (2001) J. Exp. Med. 193: 247-254

11. Lacronique V, et al. (1997) Blood 90: 3050-3056

12. Kawane K, et al. (2001) Science 292: 1546-1549

13. Ferri KF and Kroemer G. (2000) Nat. Cell Biol. 2: E63-E64

14. Sekizawa A, et al. (2000) Prenat. Diagn. 20: 886-889

15. Weil M, et al. (1998) J. Cell. Sci. 111:2707-2715

16. Berg CP, et al. (2001) Cell Death Differ. 8: 1197-1206

17. Bratosin D, et al. (2001) Cell Death Differ. 8: 1143-1156

18. Boas FE, et al. (1998) Proc. Natl. Acad. Sci. USA 95: 3077-3081

19. Romero PJ and Romero EA. (1999) Cell Calcium 26: 131-137

20. Lankiewicz S, et al. (2000) J. Biol. Chem. 275: 17064-17071

21. Blomgren K, et al. (2001) J. Biol. Chem. 276: 10191-10198

22. Shiraishi K, et al. (2000) Biol. Reprod. 63: 1538-1548
23. Squier MK, et al. (1999) J. Cell Physiol. 178: 311-319

24. Waterhouse NJ, et al. (1998) Cell Death Differ. 5: 1051-1061

25. Ruiz-Vela A, et al. (2001) J. Exp. Med. 194: 247-254

26. Wolf BB, et al. (1999) Blood 94: 1683-1692

27. Nakagawa T. and Yuan J. (2000) J. Cell Biol. 150: 887-894

28. Yamamoto AM, et al. (1998) Leukemia 12: 1467-1472

29. Gao G. and Dou QP. (2000) J. Cell. Biochem. 80: 53-72

30. Chen M, et al. (2001) J. Biol. Chem. 276: 30724-30728

31. Wang KK. (2000) Trends. Neurosci. 23: 59

32. Azam M, et al. (2001) Mol. Cell. Biol. 21: 2213-2220

33. Schulze-Osthoff K, et al. (1994) J. Cell. Biol. 127: 15-20

34. Castedo M, et al. (1996) J. Immunol. 157: 512-521

35. Susin SA, et al. (2000) J. Exp. Med.192: 571-580

36. Kroemer G. and Reed JC. (2000) Nat. Med. 6: 513-519

37. Ameisen JC, et al. (1995) Cell Death Differ. 2: 285-300

38. Madeo F, et al. (1999) J. Cell. Biol. 145: 757-767 